Division of Preventive Medicine, Brigham and Women's Hospital, Boston, MA, USA

Correspondence to: N P Paynter npaynter@partners.org

Additional material is published online only. To view please visit the journal online.

Cite this as: $B M / 2016 ; 354: i 4450$ http://dx.doi.org/10.1136/bmj.i4450

Accepted: 7 July 2016

\section{Adding tests to risk based guidelines: evaluating improvements in prediction for an intermediate risk group}

\author{
Nina P Paynter, Nancy R Cook
}

Using an additional test in patients who
are not at high enough risk of
developing disease to confidently treat, or low enough risk to confidently not treat would seem to be a straightforward way to resolve the clinical equipoise and is recommended by many guidelines. However, clear methods for evaluating the population utility of additional tests in this group have not been established. This paper includes worked examples and simulation data to show that focus on the intermediate risk group alone can be misleading and that population utility is best evaluated across the full range of risk.

Decisions about treatment attempt to best balance risks and benefits, with estimation of the risk of disease prior to treatment playing a critical role in that process at both the individual and the population level. Though there is rarely a perfect threshold of risk for action, guidelines in multiple settings have arrived at useful risk cut points to inform treatment decisions. These cut points often result in three implicit or explicit strata: risk high enough to confidently treat, risk low enough to confidently not treat, and those in between, or the "intermediate risk" group. Though at the individual level, this clinical equipoise may be resolved with a discussion between doctor and patient, from a guideline perspective, the recommendation might include subsequent testing that can appropriately reclassify people into a low risk or high risk stratum and improve prediction at a population

\section{SUMMARY POINTS}

Measures of prediction improvement in the intermediate risk group can be biased

(non-zero) when there is no true relation between the new test and the outcome

The impact of a new test on the intermediate risk group is best assessed in the context of the full population. This includes:

- Estimation of the model in which the new test is evaluated in the full population rather than the intermediate risk group alone

- Use of the full population to estimate the expected prediction improvement under the null

- Presentation of both the observed and the expected prediction improvement, or a bias correction in the case of the net reclassification improvement, in the interpretation of the overall impact

Consider a sample of the full population if a smaller study is necessary level. However, in contrast with evaluating a new marker for inclusion in the overall risk model, the process for evaluating prediction improvement in the intermediate risk group is not well developed.

\section{Case study: cardiovascular disease risk}

Risk prediction is a widely discussed tool in the prevention of cardiovascular disease and treatment of related risk factors, such as cholesterol. Current guidelines estimate risk of future cardiovascular disease events, using a risk score such as QRISK2 ${ }^{1}$ or the pooled cohort equations, ${ }^{2}$ to guide treatment decisions. The joint American College of Cardiology and American Heart Association guidelines on the treatment of cholesterol, ${ }^{3}$ use a threshold of $7.5 \% 10$ year cardiovascular disease risk to identify a subset of high risk people who might benefit from statin treatment. They also implicitly create an intermediate risk stratum of people from $5 \%$ up to $7.5 \%$ 10 year risk for potential treatment and suggest that additional factors or tests, such as family history, C reactive protein level, or coronary artery calcium score might be considered as part of individual clinician-patient discussions and decision making. Similarly, the UK National Institute for Health and Care Excellence guidelines ${ }^{4}$ use a threshold of $10 \% 10$ year risk to identify high risk people for treatment and direct clinicians to take additional factors or tests into account in treatment decisions when risk is near the threshold. The Joint British Societies' consensus recommendations ${ }^{5}$ call for additional research specifically directed at how new markers perform in the intermediate risk group.

Consequently, studies of new markers and tests have focused on improving prediction in the intermediate risk population. Some have measured the new marker in the entire population and calculated a measure of improvement for the intermediate risk group alone $\left(\mathrm{eg},{ }^{6}\right)$. Others have measured the new marker only in the intermediate risk group and based all analyses on that group alone $\left(\mathrm{eg},{ }^{7}\right)$. There have also been trials randomizing people at intermediate risk to receive additional information, such as a genetic risk score. ${ }^{8}$

We propose a strategy for research and evaluation of new markers for the intermediate risk group (see box 1). These are illustrated using results of a simulation study based on cardiovascular disease risk, as well as an example using real data, to highlight the consequences of different analytic choices. Our results are based on the assumption that the association between the outcome and the new test is no different in the intermediate risk group from that in the full population.

\section{Example with a true association}

Our first example, shown in table 1, uses a model without high density lipoprotein cholesterol as the existing 


\section{Box 1: Proposed process}

- Estimate new risk model including both new marker and traditional factors in full population and evaluate the coefficient for the new marker

- If significant, proceed to other measures of evaluation, including performance in intermediate risk group

- Present any evaluation of performance in intermediate risk group along with the expected value if there were no association to provide context

- Adjust for the expected value if conducting a test or estimating a net reclassification improvement

Table 1 Prediction measures examining the effect of adding high density lipoprotein (HDL) cholesterol to cardiovascular disease risk models in the Women's Health Study

Prediction measure

Models derived using full population

Models derived using

Ln HDL coefficient (SE), P value

$-1.0(0.14),<0.001$ intermediate risk group

Measures for changes in prediction for intermediate risk group comparing model adding HDL to model without HDL

\begin{tabular}{|c|c|c|c|}
\hline & Original & Expected under the null & \\
\hline Probability of a case moving up $(95 \% \mathrm{Cl})^{\star}$ & $0.27(0.18$ to 0.37$)$ & 0.22 & $0.45(0.35$ to 0.56$)$ \\
\hline Probability of a non-case moving up $(95 \% \mathrm{Cl})^{\star}$ & 0.20 (0.18 to 0.22$)$ & 0.17 & $0.28(0.27$ to 0.31$)$ \\
\hline Probability of a case moving down $(95 \% \mathrm{Cl})^{\star}$ & $0.14(0.09$ to 0.23$)$ & 0.19 & $0.16(0.10$ to 0.26$)$ \\
\hline Probability of a non-case moving down $(95 \% \mathrm{Cl})^{\star}$ & $0.27(0.25$ to 0.29$)$ & 0.29 & $0.25(0.23$ to 0.27$)$ \\
\hline Intermediate NRI $(95 \% \mathrm{Cl}) \dagger$ & $0.20(0.05$ to 0.33$)$ & 0.15 & 0.25 (0.10 to 0.41$)$ \\
\hline Bias corrected & $0.05(-0.10$ to 0.18$)$ & & NA \\
\hline Reclassification calibration for model without HDL (P value) & $6.7(0.04)$ & & $6.8(0.03)$ \\
\hline Reclassification calibration for model with HDL (P value) & $1.6(0.5)$ & & $0.7(0.7)$ \\
\hline
\end{tabular}

$\mathrm{NA}=$ not applicable; $\mathrm{NRI}=$ net reclassification improvement.

*Confidence intervals calculated using Agresti-Coull method.

tConfidence intervals calculated using bootstrap.

\section{Box 2: Women's Health Study example}

- The Women's Health Study is a longitudinal cohort of initially healthy women followed for incident cardiovascular disease. ${ }^{14}$ Participants provided informed consent and the study was approved by the institutional review board of Brigham and Women's Hospital. The following risk factors for cardiovascular disease have been shown to be predictive in this population: age, blood pressure, total and high density lipoprotein cholesterol, hemoglobin $\mathrm{A}_{1 \mathrm{c}}$ if diabetic at baseline, smoking, $C$ reactive protein, and family history of premature myocardial infarction ${ }^{15}$

- We used the 24558 women (560 events) with complete data on risk factors and known cardiovascular disease status at eight years for two scenarios:

- We compared a model with all risk factors except high density lipoprotein cholesterol (a known strong risk factor) with a complete model including high density lipoprotein cholesterol

- We compared the model with all the risk factors including high density lipoprotein with one adding homocysteine (a historical candidate risk factor) using a similar framework

- The reclassification used the eight year equivalents ( $<4 \%, 4 \%$ to $<6 \%$, and $\geq 6 \%$ ) of the joint American College of Cardiology and American Heart Association 10 year risk strata ( $<5 \%, 5 \%$ to $<7.5 \%, \geq 7.5 \%$ ). Models were run using the entire dataset and then rerun only in the participants with predicted intermediate risk values using the initial model (without the new marker)

score and evaluates high density lipoprotein cholesterol as a new marker in the Women's Health Study (see box 2 for additional details). As outlined in our proposed method, the first step is to estimate a risk model that includes both the new marker and the components of the established score in the full population. In such nested models, the most efficient and reliable test of independent improvement in prediction is the coefficient for the new marker. ${ }^{9}$ We evaluate the coefficient for the natural log of high density lipoprotein cholesterol from the model, which is statistically significant $(\mathrm{P}<0.001)$ when calculated in the full data. If, instead, we had used only those participants in the intermediate risk group from the established model to estimate our model, the coefficient for high density lipoprotein cholesterol would not be significant $(\mathrm{P}=0.13)$, likely due to a smaller sample size as well as a more limited range for the predictor variables.

Given a significant coefficient, the next step is to examine additional measures of clinical utility. In light of our setting of established risk strata, with new tests being considered only for those at intermediate risk, we focus primarily on measures that incorporate these risk strata. For simplicity we also focus on binary events, where the outcome is known at a specific time pointfor example, at 10 years - though many of the methods discussed have been extended to the setting of survival models.

One simple metric of change in prediction for the intermediate risk group is the probability of cases and non-cases being reassigned to the high risk or low risk groups, similar to the sensitivity and specificity of the new marker. In our example, adding high density lipoprotein to the model calculated using all the data, reclassified $27 \%$ of the initially intermediate risk cases over the threshold into high risk. However, it also reclassified $20 \%$ of the non-cases into the high risk group.

However, some movement would be expected even with a marker not associated with cardiovascular disease. Since the full range of data are available, a table of the expected changes in predicted risk if there were no association can be calculated and used to generate an estimate of the expected value for each of the prediction measures. ${ }^{10}$ We outline this method in fig 1 . Now each measure can be compared with its expected value to obtain a clearer picture of the actual improvement, as shown in table 1. For the reclassification of cases to high risk, this comparison suggests that adding high density 
The observed distribution of predicted risk in the cases is:

Model without HDL Predicted 8 year risk of CVD from model with HDL

\begin{tabular}{lccc}
\cline { 2 - 3 } & $<4 \%$ & 4 to $<6 \%$ & $<4 \%$ \\
$44 \%$ & 228 & 21 & 2 \\
4 to $<6 \%$ & 12 & 51 & 23 \\
$26 \%$ & 0 & 14 & 209
\end{tabular}

Based on this table, the probability of cases categorized as intermediate risk by the model without $\mathrm{HDL}$ moving to the high risk stratum would be $23 /(12+51+23)=0.267$. Similarly, the crude reclassification improvement $(\mathrm{RI})$ for intermediate risk cases would be $(23-12) /(12+51+23)=0.128$

For the expected table, the diagonal cells remain unchanged, and the off-diagonal cells are averaged, as shown:

Model without HDL

Predicted 8 year risk of CVD from model with HDL

\begin{tabular}{lccc}
\cline { 2 - 4 } & $<4 \%$ & 4 to $<6 \%$ & $<4 \%$ \\
$<4 \%$ & 228 & $=(21+12) / 2=16.5$ & $=(2+0) / 2=1$ \\
4 to $<6 \%$ & $=(21+12) / 2=16.5$ & 51 & $=(23+14) / 2=18.5$ \\
$26 \%$ & $=(2+0) / 2=1$ & $=(23+14) / 2=18.5$ & 209
\end{tabular}

Once the expected table is generated, it can be used to calculate the expected values for any prediction measure. For example, the expected probability of cases categorized as intermediate risk by the model without HDL moving to the high risk stratum would be $18.5 /(16.5+51+18.5)=0.215$ Similarly, the expected RI for intermediate risk cases would be $(18.5-16.5) /(16.5+51+18.5)=0.023$

Following a similar process for non-cases we start with the observed distribution of predicted risk: Model without $\mathrm{HDL}$ Predicted 8 year risk of CVD from model with HDL

\begin{tabular}{lccc}
\cline { 2 - 4 } & $<4 \%$ & 4 to $<6 \%$ & $<4 \%$ \\
4 to $<6 \%$ & 20322 & 491 & 18 \\
$\geq 6 \%$ & 417 & 840 & 308 \\
& 32 & 217 & 1353
\end{tabular}

The crude RI for intermediate risk non-cases would be $(417-308) /(417+840+308)=0.070$, resulting in a crude NRI for the intermediate risk group of $0.128+0.070=0.198$

And calculate the expected table as follows:

Model without HDL

Predicted 8 year risk of CVD from model with HDL

\begin{tabular}{ccc}
\hline$\ll 4 \%$ & 4 to $<6 \%$ & $<4 \%$ \\
20322 & $=(491+417) / 2=454$ & $=(18+32) / 2=25$ \\
$=(491+417) / 2=454$ & 840 & $=(217+308) 2=262.5$ \\
$=(18+32) / 2=25$ & $=(217+308) 2=262.5$ & 1353
\end{tabular}

$<4 \%$

4 to $<6 \%$

$=(18+32) / 2=2$

$=(217+308) 2=262.5$

1353

The expected RI for intermediate risk non-cases would be $(454-262.5) /(454+840+262.5)=0.123$, resulting in an expected NRI for the intermediate risk group of $0.023+0.123=0.146$

The results for the expected values under the null can be subtracted from the crude measures to correct the measures in the intermediate risk group. The bias corrected RI for cases is $0.128-0.023=0.105$, and the bias corrected RI for non-cases is $0.070-0.123=-0.053$ The bias corrected NRI is then $0.105-0.053(=0.198-0.146)=0.052$

Fig 1 | Calculation of the expected table of predicted risk under the null for bias correction. The expected table is based on a symmetry assumption using the whole dataset and is constructed separately for the cases and controls. We use the high density lipoprotein cholesterol example in the Women's Health Study to work through the process. HDL=high density lipoprotein; $\mathrm{CVD}=$ cardiovascular disease; $\mathrm{NRI}=$ net reclassification improvement; $\mathrm{Rl}=$ reclassification improvement higher risk stratum than to a lower risk stratum and non-cases have a higher probability of moving to a lower risk stratum than to a higher risk stratum. The same strategy can be used among those who start at intermediate risk. While the expected value for the net reclassification improvement overall is 0 if there is no association we have previously shown that substantial bias may occur if the net reclassification improvement is calculated only for the intermediate group and not corrected using the method from fig $1 .{ }^{10}$ In the full data for high density lipoprotein cholesterol, the net reclassification improvement for the intermediate risk group alone has a $95 \%$ bootstrap confidence interval (0.05 to 0.33 ) that does not include 0 , suggesting improvement in prediction. However, the $95 \%$ confidence interval for the bias corrected net reclassification improvement (observed minus expected) of $(-0.10$ to -0.18$)$ does include 0 , and the estimated effect is lower.

To compare the observed risk in each stratum to the average predicted risk, a reclassification calibration test can also be performed, with a significant $P$ value suggesting a lack of fit. ${ }^{12}$ Like the net reclassification improvement, it is usually performed on the whole table, but it can be computed in the intermediate risk subset. The regression calibration measures are also consistent with better fit in the model that includes high density lipoprotein cholesterol.

The corresponding simulation results are presented in fig 2, panel A, for a hypothetical new marker with an odds ratio of 2 for a 2 standard deviation difference. The supplemental appendix provides additional details about the simulations. The dark blue bars represent the distribution of the measures obtained if the risk model is estimated in the full population, while the white bars correspond to using only the intermediate risk group of the established score to estimate the risk model. To address the question of whether any difference is entirely due to sample size, as the intermediate risk group is inherently smaller than the full population from which it is derived, the light blue bar represents a random sample of the full population equivalent in size to the intermediate risk group, termed the scaled population. In general, the observed values are larger when the model is derived only in the intermediate risk group, where the expected values cannot be calculated.

\section{Example with no association}

Our second example, shown in table 2, uses a model without homocysteine as the existing score and evaluates homocysteine as a new marker. In this example, the coefficient for homocysteine was not significant when the full population was used for model estimation or when only the group identified as intermediate risk by the established model was used. Though this confirms the importance of using the coefficient as the initial test of association, we present all the results for discussion. For all of the measures, estimates obtained from the models in the full population are consistent with the non-significant coefficient. However, the results are noticeably different when using the participants at intermediate risk for model development. 


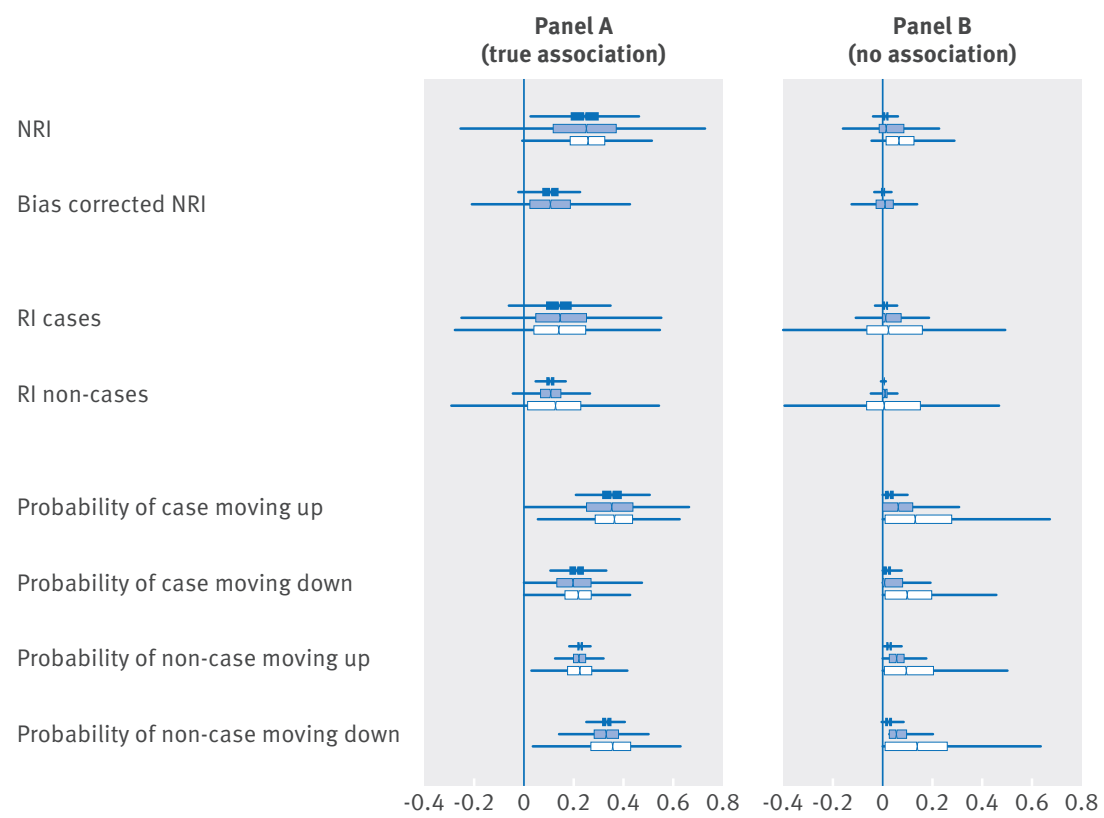

Fig 2 | Distribution of measures using cardiovascular disease cut points in a group at intermediate risk when the new marker has a true association (odds ratio of 2 for a 2 standard deviation difference) with the outcome (panel A) and no association (panel B). The boxes show the results when different populations are used to calculate the risk model with the new marker: dark blue boxes use the full population, light blue boxes use the scaled population (a random sample of the full population with the same number of participants as the intermediate risk population), and white boxes use the intermediate risk population as determined by the original model only. $\mathrm{NRI}=$ net reclassification improvement; $\mathrm{RI}=$ reclassification improvement

In this situation, the probabilities of moving and the net reclassification improvement would suggest a large improvement, and expected values under the null cannot be calculated. Supplemental table 1 presents the full reclassification table for this example.

The corresponding simulation results are presented in fig 2, panel B, for a hypothetical new marker with an odds ratio of 1 . Supplemental table 2 shows that the corresponding type 1 error rates are above $25 \%$ for the net reclassification improvement if the intermediate risk group is used for the model estimation, but that they are lower if the full population is used.
When symmetric cut points of half and twice the average risk in the population were used, all measures were less variable but the type 1 error rates were as high or higher, whereas correlations between the established factors and the new marker did not affect the results. Supplemental table 3 and the supplemental figures show these additional results.

Many other excellent measures of prediction exist, including the difference in the $\mathrm{C}$ statistic, the integrated discrimination improvement, and continuous net reclassification improvement, among others. These measures are an important part of the overall presentation and should be incorporated when evaluating the risk prediction performance of a new marker. In supplemental table 4 we have included our simulation results for the rate of type 1 errors for these measures when estimating the model with the new marker and the established risk factors in the intermediate risk group alone instead of the full population. Though the effect sizes are small, the rate of type 1 errors does increase if only the intermediate risk group is used for the integrated discrimination improvement and continuous net reclassification improvement, showing the same pattern as the categorical markers. The difference in the $\mathrm{C}$ statistic, on the other hand, is overly conservative in the intermediate group, as has been observed in other settings. ${ }^{13}$

\section{Conclusions}

Measures of model improvement may be biased when based just on the intermediate risk group. Recommendations for additional testing, even when the intermediate risk group is of primary interest, should be based on research conducted across the full spectrum of risk. This efficient design provides a more stable measure of improvement in the intermediate risk group when there is clinical justification for using the new test in the intermediate risk group only and the independent effect of the marker has been demonstrated. It also allows for reanalysis in response to changes in cut points as well as the possibility of exploring improvements in prediction in other groups. Additionally, the effect size of all prediction measures in the intermediate risk group should be

\section{Table 2 | Prediction measures examining the effect of adding homocysteine to cardiovascular disease risk models in Women's Health Study}

\begin{tabular}{|c|c|c|c|}
\hline Prediction measure & \multicolumn{2}{|c|}{ Models derived using full population } & $\begin{array}{l}\text { Models derived using } \\
\text { intermediate risk group }\end{array}$ \\
\hline Ln homocysteine coefficient (SE), P value & $0.18(0.11), 0.10$ & & $0.44(0.28), 0.12$ \\
\hline \multicolumn{4}{|c|}{ Measures for changes in prediction for the intermediate risk group comparing model adding homocysteine to model without homocysteine } \\
\hline & Original & Expected und & \\
\hline Probability of a case moving up $(95 \% \mathrm{Cl})^{\star}$ & $0.03(0.01$ to 0.10$)$ & 0.05 & $0.36(0.27$ to 0.47$)$ \\
\hline Probability of a non-case moving up $(95 \% \mathrm{Cl})^{\star}$ & $0.04(0.03$ to 0.05$)$ & 0.04 & $0.27(0.25$ to 0.30$)$ \\
\hline Probability of a case moving down $(95 \% \mathrm{Cl})^{*}$ & $0.06(0.02$ to 0.13$)$ & 0.05 & $0.08(0.04$ to 0.16$)$ \\
\hline Probability of a non-case moving down $(95 \% \mathrm{Cl})^{\star}$ & $0.06(0.05$ to 0.07$)$ & 0.29 & $0.25(0.23$ to 0.27$)$ \\
\hline Intermediate NRI (95\% Cl)† & -0.01 (0.0 to 0.33$)$ & 0.06 & $0.20(0.07$ to 0.33$)$ \\
\hline Bias corrected & \multicolumn{2}{|l|}{$0.02(-0.10$ to 0.18$)$} & NA \\
\hline Reclassification calibration for model without homocysteine (P value) & \multicolumn{2}{|l|}{$1.2(0.6)$} & $7.5(0.02)$ \\
\hline Reclassification calibration for model with homocysteine (P value) & \multicolumn{2}{|l|}{$1.5(0.5)$} & $2.0(0.4)$ \\
\hline
\end{tabular}


presented in the context of the expected value under the null or bias corrected to avoid over-optimism.

Contributors: NP and NC contributed to the design, concept, and interpretation. NP carried out the analysis and drafted the manuscript. NC provided critical revisions. NP is the guarantor.

Funding: This project was supported by grant HL113080 from the National Heart, Lung, and Blood Institute. The funder had no role in the study design, analysis, or reporting.

Competing interests: All authors have completed the ICMJE uniform disclosure form at www.icmje.org/coi disclosure.pdf and declare: NP and NC are supported by the National Heart Lung and Blood Institute; no financial relationships with any organizations that might have an interest in the submitted work in the previous three years; no other relationships or activities that could appear to have influenced the submitted work.

1 Hippisley-Cox J, Coupland C, Vinogradova Y, et al. Predicting cardiovascular risk in England and Wales: prospective derivation and validation of QRISK2, 2008.

2 Goff DC Jr, , Lloyd-Jones DM, Bennett G, et al. American College of Cardiology/American Heart Association Task Force on Practice Guidelines. 2013 ACC/AHA guideline on the assessment of cardiovascular risk: a report of the American College of Cardiology/ American Heart Association Task Force on Practice Guidelines. J Am Coll Cardiol 2014:63(25 Pt B):2935-59. doi:10.1016/j.jacc.2013.11.005.

3 Stone NJ, Robinson JG, Lichtenstein AH, et al. American College of Cardiology/American Heart Association Task Force on Practice Guidelines. 2013 ACC/AHA guideline on the treatment of blood cholesterol to reduce atherosclerotic cardiovascular risk in adults: a report of the American College of Cardiology/American Heart Association Task Force on Practice Guidelines. J Am Coll Cardiol 2014:63(25 Pt B):2889-934 doi:10.1016/j.jacc.2013.11.002

4 National Institute for Health and Care Excellence. Cardiovascular disease: risk assessment and reduction, including lipid modification NICE guidelines [CG181], 2014.

5 Boon N, Boyle R, Bradbury K, et al. JBS3 Board. Joint British Societies' consensus recommendations for the prevention of cardiovascular disease (JBS3). Heart 2014;100(Suppl 2):ii1-67. doi:10.1136/ heartjinl-2014-305693.
6 Würtz P, Havulinna AS, Soininen P, et al. Metabolite profiling and cardiovascular event risk: a prospective study of 3 population-based cohorts. Circulation 2015:131:774-85. doi:10.1161/

CIRCULATIONAHA.114.013116.

7 Yeboah J, McClelland RL, Polonsky TS, et al. Comparison of novel risk markers for improvement in cardiovascular risk assessment in intermediate-risk individuals. JAMA 2012;308:788-95. doi:10.1001/ jama.2012.9624.

8 Kullo IJ, Jouni H, Olson JE, Montori VM, Bailey KR. Design of a randomized controlled trial of disclosing genomic risk of coronary heart disease: the Myocardial Infarction Genes (MI-GENES) study BMC Med Genomics 2015;8:51. doi:10.1186/s12920-015-0122-0.

9 Pepe MS, Kerr KF, Longton G, Wang Z. Testing for improvement in prediction model performance. Stat Med 2013;32:1467-82. doi:10.1002/sim.5727.

10 Paynter NP, Cook NR. A bias-corrected net reclassification improvement for clinical subgroups. Med Decis Making 2013:33:154 62. doi:10.1177/0272989X12461856

11 Pencina MJ, D’Agostino RBS Sr, D’Agostino RBJ Jr, Vasan RS. Evaluating the added predictive ability of a new marker. from area under the ROC curve to reclassification and beyond. Stat Med 2008;27:157-72, discussion 207-12. doi:10.1002/sim.2929

12 Cook NR, Paynter NP. Performance of reclassification statistics in comparing risk prediction models. Biom J 2011;53:237-58. doi:10.1002/bimj.201000078

13 Demler OV, Pencina MJ, D’Agostino RB Sr. Misuse of DeLong test to compare AUCs for nested models. Stat Med 2012;31:2577-87. doi:10.1002/sim.5328

14 Ridker PM, Cook NR, Lee IM, et al. A randomized trial of low-dose aspirin in the primary prevention of cardiovascular disease in women. NEngl / Med 2005:352:1293-304 doi:10.1056/NEIMoa050613.

15 Ridker PM, Buring JE, Rifai N, Cook NR. Development and validation o improved algorithms for the assessment of global cardiovascular risk in women: the Reynolds Risk Score. JAMA 2007;297:611-9. doi:10.1001/jama.297.6.611

(c) BMJ Publishing Group Ltd 2016

Supplemental file: supplemental tables 1-4 and figures 1 and 2 